\title{
Updates and Best Practices in the Management of Facial Erythema
}

\author{
Jameson Loyal ${ }^{1}$ \\ Emily Carr ${ }^{2}$ \\ Rawaa Almukhtar ${ }^{2}$ \\ Mitchel P Goldman ${ }^{2,3}$ \\ 'Department of Dermatology, State \\ University of New York Downstate \\ Health Sciences University, Brooklyn, NY, \\ USA; ${ }^{2}$ Cosmetic Laser Dermatology: \\ A West Dermatology Company, San \\ Diego, CA, USA; ${ }^{3}$ Department of \\ Dermatology, University of California, \\ San Diego, CA, USA
}

\begin{abstract}
Facial erythema is a common dermatologic complaint. There are many medical and procedure-based treatments to help reduce the appearance of unwanted facial redness. The authors review a variety of treatment options and techniques to reduce facial erythema and prominent facial veins including topical medical therapies, a variety of lasers, light- and energy-based devices as well as the use of neuromodulators and sclerotherapy. The benefits and potential pitfalls of each procedure modality are also highlighted.
\end{abstract}

Keywords: facial redness, flushing, rosacea, facial vessels, lasers, energy-based devices

\section{Introduction}

Facial erythema may be a component of a variety of clinical entities, including rosacea, post-inflammatory erythema from inflammatory dermatoses and scars, keratosis pilaris, actinic photodamage, acne, folliculitis, seborrheic dermatitis, periorificial dermatitis, eczematous dermatitides, episodic flushing, lupus erythematosus, and photosensitive/photoallergic eruptions, and many more. A thorough clinical examination is necessary to make the correct diagnosis to ensure the most appropriate and effective treatment modality. There are numerous treatment options available, including medical and/or laser, light and energy-based devices, that can ameliorate unwanted facial redness, telangiectasias, and superficial veins. We review multiple therapies that can broaden one's armamentarium when treating a patient with diffuse facial erythema. Particular attention will be paid to energybased devices as well as combination treatments. Additionally, there will be particular focus on the treatment of the prototypic disorder presenting with facial erythema, rosacea, which can guide treatment for other diagnoses presenting with unwanted erythema.

\section{Background Information and Impact}

Rosacea, with an approximate global prevalence of $5.46 \%$ among the adult population, is one of the most common diagnoses that prompts a patient to seek evaluation for unwanted facial erythema and visible telangiectasias. ${ }^{1}$ If untreated, facial erythema can have serious psychological and social consequences as well as physical disfigurement. ${ }^{2}$ Moreover, patients with rosacea are more likely to be depressed, have social phobias, and have an overall higher perception of their disease. $^{2}$ The decrease in quality of life has been demonstrated in a variety of studies using validated questionnaires including the Dermatology Life Quality Index (DLQI) and the Rosacea Quality of Life Index (RosaQoL). Importantly,
Correspondence: Jameson Loyal Cosmetic Laser Dermatology: A West Dermatology Company, 9339 Genesee Avenue, Suite \#300, San Diego, CA, 92121 , USA

Tel + I 858 943-2 II3

Email Jameson.Loyal@gmail.com 
treatment of diffuse facial erythema has been demonstrated to improve a patient's psychological symptoms as well as quality of life. ${ }^{2}$

It is paramount that the clinician differentiate perilesional erythema stemming from inflammatory lesions, more commonly seen in the papulopustular subtype, from diffuse background erythema. ${ }^{3}$ Additionally, background erythema should be differentiated between transient flushing and persistent erythema. ${ }^{4}$ Treatment of the inflammatory papules with a variety of topical and oral therapies will aid in the resolution of not only the papules but also to some extent the perilesional erythema. ${ }^{3}$ Background erythema; however, is usually recalcitrant to therapies aimed at inflammatory papules and more difficult to treat with medical therapy alone.

Diffuse and persistent facial erythema can be seen in all types of rosacea but is most classically described in the erythematotelangiectatic subtype. ${ }^{3}$ The erythema is the result of dilation of cutaneous blood vessels and increased blood flow to the skin. ${ }^{5}$ This type of diffuse erythema is usually macular and/or slightly edematous, confluent, and bilaterally symmetric. ${ }^{3}$ Involvement of the central face is classic with the medial cheeks, nose, chin, and midforehead most accentuated but involvement of the lateral face may also occur. ${ }^{3}$ Intensity of the erythema can be exaggerated during flares; however, variable baseline erythema will be present during periods of disease quiescence. ${ }^{3}$ Telangiectasias are commonly seen on the medial cheeks, nose, and perinasal regions. ${ }^{3}$ Although the underlying mechanism of this erythema is still under investigation, current research points to an augmented innate immune response, neurovascular dysregulation, and nitric oxide and cathelicidin peptides resulting in provocation of vasodilation of vascular smooth muscle and inflammation. ${ }^{2,3,6}$ Additionally, neovascularization and angiogenesis over time leads to the formation of telangiectasias. $^{3}$

Moreover, impairment of the stratum corneum barrier, depletion of cutaneous antioxidants, and overall photodamage from ultraviolet radiation can exacerbate any underlying primary cutaneous pathology causing diffuse facial erythema. ${ }^{3}$ The clinician should ensure optimization of the skin barrier function during all consultations for unwanted facial erythema. This is especially important because a damaged skin barrier allows for excessive transepidermal water loss and entry of allergens which to susceptible patients can exacerbate underlying skin pathologies such as rosacea and eczematous dermatitides, to name a few. Additionally, degradation of the skin barrier, will leave skin dehydrated resulting in a dull appearance and allow for the accentuation of unwanted redness. Finally, investigating potential triggers which may be exacerbating a patient's facial erythema and offering ways to alleviate these triggers such as alcohol, caffeine, ultraviolet exposure, heat exposure, et cetera can add value to a patient's treatment plan. ${ }^{4}$

\section{Methods}

The authors conducted a PubMed database search for randomized controlled trials, cohort studies, case series, and select case reports involving treatments for facial erythema, telangiectasias, and facial veins including topical medical therapies, neuromodulators, lasers, as well as light- and energy-based devices. The following keywords were used for the search: facial erythema, telangiectasias, facial veins, reticular veins, rosacea, medical therapy, brimonidine, oxymetazoline, neuromodulator, botulinum toxin, pulsed dye laser, KTP laser, Nd:YAG laser, picosecond laser, fractionated laser, IPL, microneedling, radiofrequency, radiofrequency microneedling, sclerotherapy, phlebectomy, photodynamic therapy, and Demodex. Exclusion criteria included articles not involving one of the aforementioned treatment modalities, treatment of conditions other than facial erythema, telangiectasias and facial veins, and articles not written in the English language. Descriptive summaries of these studies are presented in this review.

\section{Topical Medical Therapies}

Brimonidine $0.33 \%$ topical gel (Mirvaso $^{\circledR}$, Galderma Laboratories, L.P., Fort Worth, Texas, USA) and oxymetazoline $1 \%$ cream (Rhofade ${ }^{\mathrm{TM}}$, EPI Health, LLC., Charleston, South Carolina, USA) are two topical therapies that were approved for once daily use by the Food and Drug Administration (FDA) in 2013 and 2017, respectively, for the treatment of persistent facial erythema in adult patients with rosacea. The benefit of these topicals is the ability to offer patients an as-needed option for those seeking a short-term fix for a special event but also a longterm option for those using the product daily. ${ }^{6}$

Brimonidine is a selective alpha- 2 adrenergic agonist that binds the alpha- 2 receptors on vessels resulting in vasoconstriction of small arteries and veins thereby reducing the effects of vasodilation, namely erythema. 6,7 Brimonidine can be used as monotherapy or in combination with other modalities. Interestingly, topical 
brimonidine has been proven effective in reducing treatment-related erythema from devices such as intense pulsed light (IPL). ${ }^{8}$ Peak benefit is between 3 and 6 hours after application, but patients can anticipate some reduction in facial erythema as early as 30 minutes after application. ${ }^{6}$ Erythema reduction persists for approximately 12 hours. ${ }^{6}$ The most common adverse effects include flushing, irritation, pruritus, and worsening of baseline rosacea. ${ }^{6}$ A paradoxical erythema where one can experience worsening redness within 3-6 hours of application can occur but resolves with discontinuation of use. ${ }^{9,10}$ Additionally, an exaggerated rebound erythema occurring approximately 12 hours after application has been reported in approximately $20 \%$ of patients in post-marketing surveillance data. ${ }^{6,11}$ This rebound erythema is typically worse than experienced at baseline but resolves in 6-12 hours. ${ }^{6}$ Contact dermatitis has also been rarely reported. ${ }^{6}$

Oxymetazoline binds selectively to alpha-1A receptors resulting in vasoconstriction of cutaneous vessels. ${ }^{11}$ It also has some partial affinity for the alpha-2 adrenergic receptors. ${ }^{7}$ Oxymetazoline, similar to brimonidine, only has an effect on vessels with smooth muscle and as such will not have an effect on smaller blood vessels, capillaries, and telangiectasias. ${ }^{11}$ Interestingly, there is evidence that oxymetazoline also inhibits neutrophil phagocytosis and oxidative burst thereby reducing the inflammatory milieu present in rosacea. ${ }^{11}$ An observable reduction in erythema is achieved within 1-3 hours of application and typically lasts up to 12 hours. ${ }^{7,11}$ The most common adverse effects are worsening inflammatory lesions, application site dermatitis, pruritus, and erythema. ${ }^{11}$ Unlike brimonidine, there was no clinically meaningful rebound erythema experienced by subjects using oxymetazoline for one year. ${ }^{3}$ Use of oxymetazoline as adjunctive therapy with potassium-titanyl-phosphate (KTP) lasers, 595nm pulse dye laser (PDL), and IPL has been demonstrated to be well tolerated and efficacious in further helping to reduce facial erythema. ${ }^{12,13}$

Given that brimonidine and oxymetazoline are alphaadrenergic vasoconstrictors the prescriber should be mindful of the theoretical risks associated with increased blood vessel tone especially in individuals with cardiovascular diseases and vascular insufficiency. ${ }^{14}$ There is also a potential risk of angular closure glaucoma in those with narrow angle glaucoma. ${ }^{14}$ Oxymetazoline is not advised for patients who are taking monoamine oxidase inhibitors; however, the amount and significance of any systemic absorption is not well established. ${ }^{10}$ Applying topical alpha-adrenergic vasoconstrictors on abraded or denuded skin should be used with caution due to risk of systemic absorption and an exaggerated vasoconstrictive effect. As with any pharmaceutical, the prescriber should complete a thorough history and physical examination and discuss the risks and benefits of treatment.

For all patients with diffuse facial erythema, a comprehensive skincare regimen that focuses on repairing or maintaining a healthy skin barrier, repletion of antioxidant reserves, and protection from ultraviolet radiation is paramount and should be included in every consultation for this common dermatologic complaint. ${ }^{3} \mathrm{We}$ individualize the skincare regimen with product categories that can help with revitalizing the skin including a gentle facial cleanser, antioxidant serum, a hydrating moisturizer with ingredients such as ceramides, glycerin, dimethicone, or hyaluronic acid, and a broad-spectrum mineral-based sunscreen. Moisturizers and serums with these ingredients replenish and repair the skin's barrier function by preventing excessive transepidermal water loss thereby reducing skin dehydration and preventing dry, irritated skin.

\section{Neuromodulators}

An unconventional treatment for recalcitrant facial flushing is use of botulinum toxin injected subdermally. ${ }^{15-17}$ Although there are only a few case reports demonstrating this effect, most in combination with pulsed dye laser, this treatment can potentially be useful for discrete areas of residual diffuse flushing and erythema. ${ }^{15-18}$ Practitioners should be judicious with the dilution, dosing, and placement of the botulinum toxin, paying particular attention to the underlying facial musculature and subsequent muscle paralysis that will ensue. Bloom et al used abobotulinumtoxinA in their pilot study and noted this specific neuromodulator was chosen because of its greater diffusion and migration ability as studies using other neuromodulators such as onabotulinumtoxinA were not as efficacious. ${ }^{17}$ Importantly, Bloom et al highlighted that higher doses of neuromodulators were not associated with superior results in reduction of erythema. ${ }^{17} \mathrm{We}$ recommend injecting $1 \mathrm{U}$ of neuromodulator intradermally every $1 \mathrm{~cm} .{ }^{19}$

It should be highlighted that well-designed controlled trials thoroughly investigating this treatment are needed to better understand how this treatment could benefit patients as a standalone treatment or in combination with wellestablished options. It is established that botulinum toxin inhibits acetylcholine; however, theories regarding the role 
acetylcholine plays in vasodilation of vessels and the potential of botulinum toxin in the stabilization of mast cells needs to be further elucidated. ${ }^{16}$ Finally, this newer therapeutic option could potentially find its place in combination treatment with lasers and light-based devices. ${ }^{19}$ Lasers and other light-based devices are gold standard treatments for erythema but are not optimal in controlling intermittent flushing episodes. ${ }^{16}$

\section{Lasers}

\section{Pulsed Dye Laser (PDL)}

PDL with wavelengths of 585 nanometer $(\mathrm{nm})$ or $595 \mathrm{~nm}$ and using the principle of selective photothermolysis has been the gold standard treatment for small caliber, superficial blood vessels since this laser's introduction into clinical practice in $1986 .{ }^{20}$ PDL is a reliable and standardof-care choice when it comes to procedural-based treatment for areas of erythema and telangiectasia by targeting oxyhemoglobin. Early studies using a 450msec pulsed $585 \mathrm{~nm}$ PDL found that blood vessels with a diameter greater than $0.2 \mathrm{~mm}$ require multiple treatments and vessels with diameters greater than $0.4 \mathrm{~mm}$ are not as adequately treated. $^{21}$ This laser is not the appropriate choice for targets that are greater than $1-2 \mathrm{~mm}$ in depth. ${ }^{21}$ The use of epidermal cooling also allows the operator to use higher fluences. $^{21}$ Patients often need multiple treatments and subsequent maintenance treatments to have a sustained benefit. We have found treatments to be needed every 4-6 months. Additionally, the laser surgeon must balance clearance of the target with side effects, keeping in mind acceptability of postoperative purpura. ${ }^{20}$ Short pulse durations undoubtedly result in post-treatment purpura which can last between 7 and 14 days, unacceptable to many patients. $^{22}$ Gentler, nonpurpuric settings are less efficacious and may require many more treatments compared with more aggressive settings. ${ }^{20}$ Newer PDLs with longer pulse durations and more uniform cooling allow purpurafree results. ${ }^{23-26}$ The utilization of the serial passes and/or pulse stacking techniques are needed for stubborn telangiectasias without a significant increase in adverse effects. $^{23,25}$ One should be mindful of the increased risk of postoperative hyperpigmentation and even hypopigmentation when treating patients of darker skin tones, using higher fluences, and making sure to use adequate epidermal cooling. $^{20,22}$

Pain associated with PDL treatments can be a limiting factor for some patients. The pain level of PDL treatments has been described as higher than the pain with a typical intradermal injection; however, some practitioners are hesitant to use topical anesthetics due to the potential of vasodynamic effects on blood vessels and subsequent loss of efficacy with the laser treatment. ${ }^{5}$ Chunharas et al, in a retrospective study, demonstrated that erythema scores and PDL efficacy in the patient group who had either $7 \%$ lidocaine $/ 7 \%$ tetracaine or $23 \%$ lidocaine $/ 7 \%$ tetracaine ointment applied to the treatment area one hour prior to laser treatment was statistically non-inferior to the group of patients who were not provided pretreatment anesthesia. ${ }^{5}$

Tan et al demonstrated statistically significant reduction in erythema in patients diagnosed with facial erythema secondary to rosacea when treated with two PDL sessions with purpuric settings (595nm, 9.5 to $11.5 \mathrm{~J} / \mathrm{cm}^{2}, 7$ millimeter $(\mathrm{mm})$ spot size, and 1.5 millisecond (ms) pulse duration). ${ }^{20}$ This study specifically showed an approximately $36 \%$ to $53 \%$ reduction in erythema scores depending on the area of the face and, equally as important, showed a $75.6 \%$ decrease in the Dermatology Life Quality Index (DLQI) correlating with improvement of quality of life. ${ }^{20}$ Similarly, Clark et al demonstrated in a prospective study a $50 \%$ reduction in erythema, a 55\% reduction in flushing, and a 75\% reduction in telangiectasia scores after a mean of three PDL treatments in twelve subjects. ${ }^{27}$ Common side effects included bruising which occurred in all patients and subsequently resolved in 7-10 days, post-inflammatory hyperpigmentation occurring in more than half of patients, crusting in two patients, and slight atrophic scarring in two patients, one of whom sustained crusting after the treatment. The post-inflammatory hyperpigmentation resolved after three months in all but one patient with a history of melasma. ${ }^{27}$ It was highlighted that telangiectatic areas had the greatest chance of clearance but there were no obvious clinical indicators to suggest which patients would benefit most from PDL. ${ }^{27}$

In a retrospective study, Baskan et al demonstrated that more than $60 \%$ of patients enrolled who had between 1 and 4 treatments for unwanted erythema and telangiectasias had a greater than $50 \%$ clinical improvement according to the physicians' clinical assessment with minimal transient side effects. $^{28}$ In a study investigating treatment of facial telangiectasias and erythema with either a single purpuric pass versus four subpurpuric passes using a long-pulsed PDL, Iyer et al demonstrated slightly greater reduction (43.3\%) in surface area covered by telangiectasias for patients treated with a single purpuric pass $\left(12 \mathrm{~J} / \mathrm{cm}^{2}, 7 \mathrm{~mm}\right.$ spot size, $6 \mathrm{~ms}$ pulse 
width, and $20 \mathrm{~ms}$ cryogen cooling spray with a $10 \mathrm{~ms}$ delay) compared with four subpurpuric passes $\left(6 \mathrm{~J} / \mathrm{cm}^{2}, 10 \mathrm{~mm}\right.$ spot size, $6 \mathrm{~ms}$ pulse width and $10 \mathrm{~ms}$ cryogen cooling spray with a $10 \mathrm{~ms}$ delay) $(35.9 \%) .{ }^{25}$ Interestingly, the authors also commented that the nonpurpuric treatment modality yielded more consistent results, although slightly less effective, than the single purpuric modality. ${ }^{25}$ It was also demonstrated that background erythema was better treated with the multiple pass approach with an overall reduction of erythema of $32.3 \%$ compared with a $3 \%$ change in the side of the face treated with one high-intensity pass. ${ }^{25}$ Iyer et al hypothesized that induction of purpura and vessel rupture with laser settings that are too zealous can have proinflammatory effects that could counteract the intended erythema reduction. ${ }^{25}$

Alam et al compared PDL with a microsecond $1064 \mathrm{~nm}$ Neodymium:yttrium aluminium garnet (Nd:YAG) laser for the treatment of diffuse erythema in a split-face randomized study and found that the PDL reduced facial erythema $6.4 \%$ more from baseline than did the Nd:YAG laser, after four treatments, as determined by spectrophotometer readings. ${ }^{29}$ Additionally, study subjects rated improvement of redness by a mean of $52 \%$ for the side of the face treated with PDL compared to a mean improvement of $34 \%$ on the side treated with Nd:YAG. ${ }^{29}$ Patients were more satisfied with the results of the PDL but noted slightly more pain than from the Nd:YAG. ${ }^{29}$
When treating patients with rosacea, we pass over the entire area with $50-70 \%$ overlap with subsequent passes to increase vessel clearance without purpura. Our clinical endpoint is to see the vessels blanch. Reduction of Demodex densities on the skin has been demonstrated in as little as one treatment with PDL. ${ }^{30}$ We recommend our rosacea patients undergo at least two maintenance PDL treatments per year to minimize resurgence of Demodex (Figure 1).

\section{Potassium-Titanyl-Phosphate (KTP)}

KTP lasers produce wavelengths at $532 \mathrm{~nm}$ and are vascular-specific with their wavelength corresponding with the first absorption peak of hemoglobin at $542 \mathrm{~nm} .^{22,31}$ KTP lasers are good at treating discrete, larger-caliber telangiectasia that can be visualized; however, due to their shorter wavelength they have a limited depth of penetration compared to longer wavelength lasers. ${ }^{20,31}$ In addition, the $532 \mathrm{~nm}$ wavelength has a higher coefficient of absorption for melanin which can result in unwanted pigmentary change and epidermal injury compared to other lasers used for vascular targets. ${ }^{31}$ However, even though the KTP laser has a higher coefficient of absorption for melanin than PDLs, the coefficient of absorption for oxyhemoglobin for KTPs is even greater thus lowering the potential risk of epidermal damage. ${ }^{23}$ Additionally, KTP lasers with the appropriate cooling

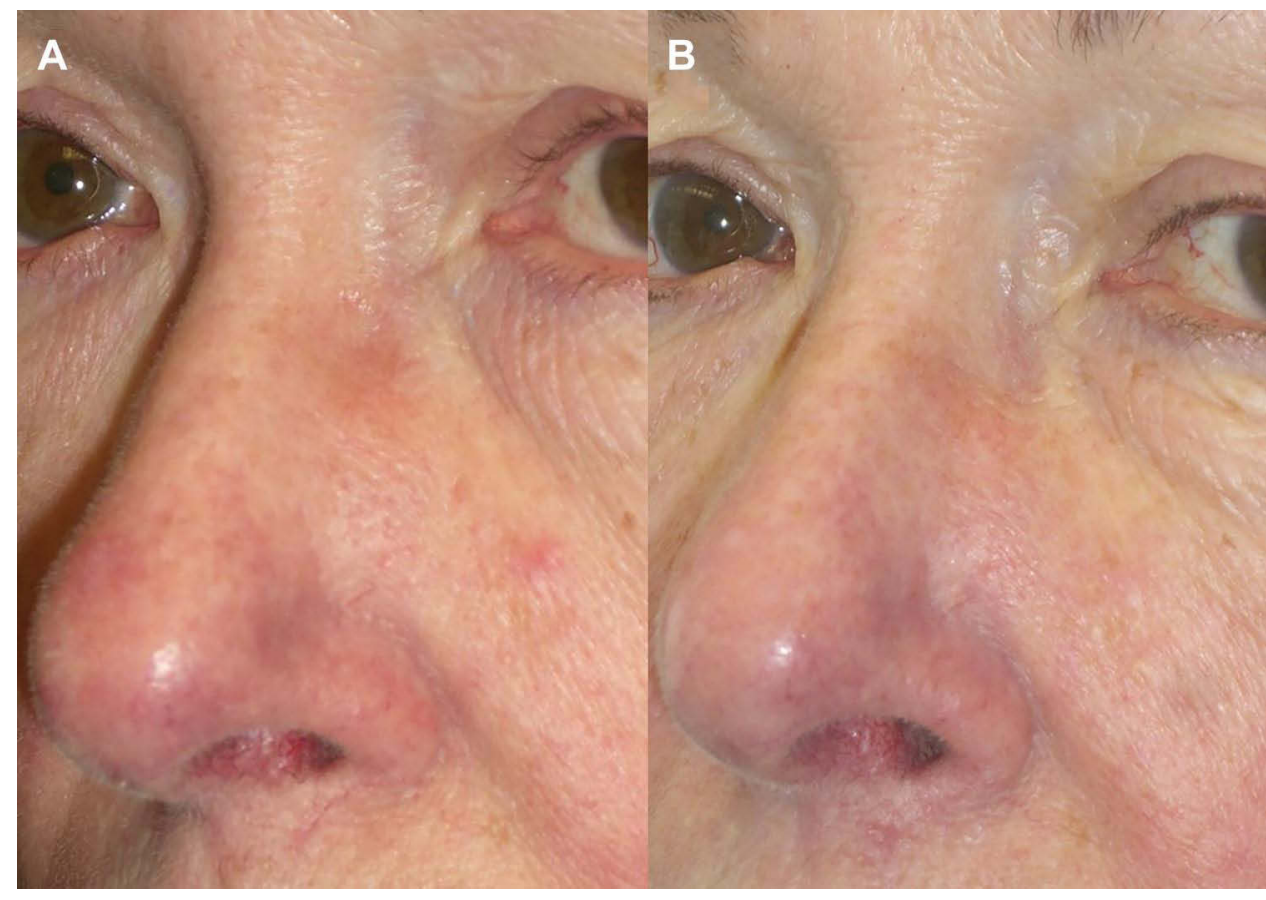

Figure I Before (A) and post one year (B) following 2 PDL treatments for erythema of the nose. Figure courtesy of Dr. Goldman. 
have demonstrated an added benefit of treating unwanted lentigines. $^{23}$ This added bonus can be extremely beneficial to patients wanting overall photorejuvenation in an efficient and cost-effective manner without the need to use a variety of different lasers. Advances in contact cooling, larger spot sizes, and greater fluences have made newer generation KTP lasers more efficient and safer. $^{23}$

This laser has not been as well validated for the treatment of diffuse erythema as has PDL; however, in a small randomized, split-face study by Uebelhoer et al, comparing the $532 \mathrm{~nm}$ KTP laser with the 595nm long-pulsed PDL for treatment of telangiectasias and diffuse facial erythema, it demonstrated an $85 \%$ improvement of erythema on the KTP treated side compared to a $75 \%$ improvement of erythema on the PDL treated side after three treatments. KTP settings for the majority of patients were $10 \mathrm{~J} / \mathrm{cm}^{2}$ at $18 \mathrm{~ms}$ and spot size of $5 \mathrm{~mm}$ treating the more prominent vessels and a single pass over the treatment area with $9 \mathrm{~J} / \mathrm{cm}^{2}$ at $23 \mathrm{~ms}$ with $10 \mathrm{~mm}$ spot size. PDL settings were a $10 \mathrm{~mm}$ spot size, a fluence of $7.5 \mathrm{~J} /$ $\mathrm{cm}^{2}$, a 10ms pulse width, and dynamic cooling settings of $30 \mathrm{~ms}$ spray and a $20 \mathrm{~ms}$ delay. ${ }^{23}$ In this study, the PDL took three treatments to obtain similar results to the KTP laser in only two sessions. ${ }^{23}$ Study subjects noted the KTP treated side cleared facial redness better than PDL after every treatment. $^{23}$ Fifty-eight percent of subjects reported a greater degree of post-treatment erythema with the KTP laser compared to PDL. ${ }^{23}$ In a large retrospective study, Becher et al demonstrated $83 \%$ of patients undergoing KTP laser treatment for telangiectasias and facial erythema had a marked improvement or better from baseline. ${ }^{26}$ However, only $33 \%$ of patients with only facial erythema demonstrated marked improvement or better from baseline, which the authors suggest is due to the pulse length of the KTP laser being too long to match the thermal relaxation time of the tiny vessels producing the facial erythema. ${ }^{26}$

Keaney et al demonstrated that the 532nm KTP laser was as safe and effective as PDL for the treatment of erythematous surgical scars. ${ }^{20,31}$ Subjects noted that the KTP laser was slightly more painful and resulted in increased erythema and edema post-treatment compared to PDL. ${ }^{31}$

\section{Neodymium:Yttrium Aluminium Garnet (Nd:YAG)}

Microsecond, millisecond, and nanosecond (Q-switched) $\mathrm{Nd}$ :YAG lasers produce wavelengths at $1064 \mathrm{~nm}$, its chromophore is hemoglobin and deoxyhemoglobin. ${ }^{21,32}$ This laser can reach depths of 4-6mm and can treat much larger vessels with diameters in the $1-3 \mathrm{~mm}$ range; however, as higher fluences and larger spot sizes are used there is an increased risk of excessive pain and dermal damage. ${ }^{21,32}$ This laser is particularly good at treating thicker and bigger vessels with clinically blue-colored hues. ${ }^{33}$ Treatment of smaller vessels often requires high energy and short pulse durations which increase the risk of purpura as well as blistering and scarring. ${ }^{32,34}$ Appropriate skin cooling is imperative to prevent scarring especially when treating deeper vessels that require higher energy. ${ }^{32,35}$ One benefit of Nd:YAG lasers is the ability to safely treat patients with darker skin complexions mainly due to the minimal absorption of melanin. ${ }^{34}$ Since this laser can be used for permanent hair reduction, patients must be notified of this effect when treating vessels in hair bearing areas.

In a split-face study, Salem et al compared three treatments of either Nd:YAG or PDL for treatment of erythema and telangiectasias and found both to be effective; however, statistical significance was not achieved. ${ }^{36}$ The authors reported more patients having side effects related to treatment-induced purpura from PDL and none from use of the Nd:YAG laser. ${ }^{36}$

Some laser devices combine both PDL and Nd:YAG technology, allowing the firing of the PDL component milliseconds prior to the Nd:YAG wavelength. ${ }^{37}$ Some believe that this technique enhances the effectiveness of the Nd:YAG laser by a factor of 3-5 due in part to the transformation of oxyhemoglobin to methemoglobin by the PDL. ${ }^{37}$ There has also been a suggestion of reduced purpura in the combined device compared to PDL alone when treating blood vessels. ${ }^{38}$ Karsai et al demonstrated, in a split-face randomized study, that use of the dual wavelength PDL-Nd:YAG delivered higher and statistically significant rates of telangiectasia clearance in one treatment that use of a single wavelength PDL or $\mathrm{Nd}$ : YAG. ${ }^{38}$ The Nd:YAG laser has also been used successfully in the eradication of arterioles on the nasal ala and tip which can be quite recalcitrant to treatment with either PDL or IPL. ${ }^{39}$ However, treating large vessels in the nasal alar crease requires high energies so epidermal cooling is important to prevent excessive non-specific thermal damage resulting in hypopigmentation and/or epidermal atrophy (Figure 2). There are even reports that the $\mathrm{Nd}$ : YAG laser can lead to decreased Demodex densities in individuals with rosacea, which can further improve this condition. $^{40}$ 


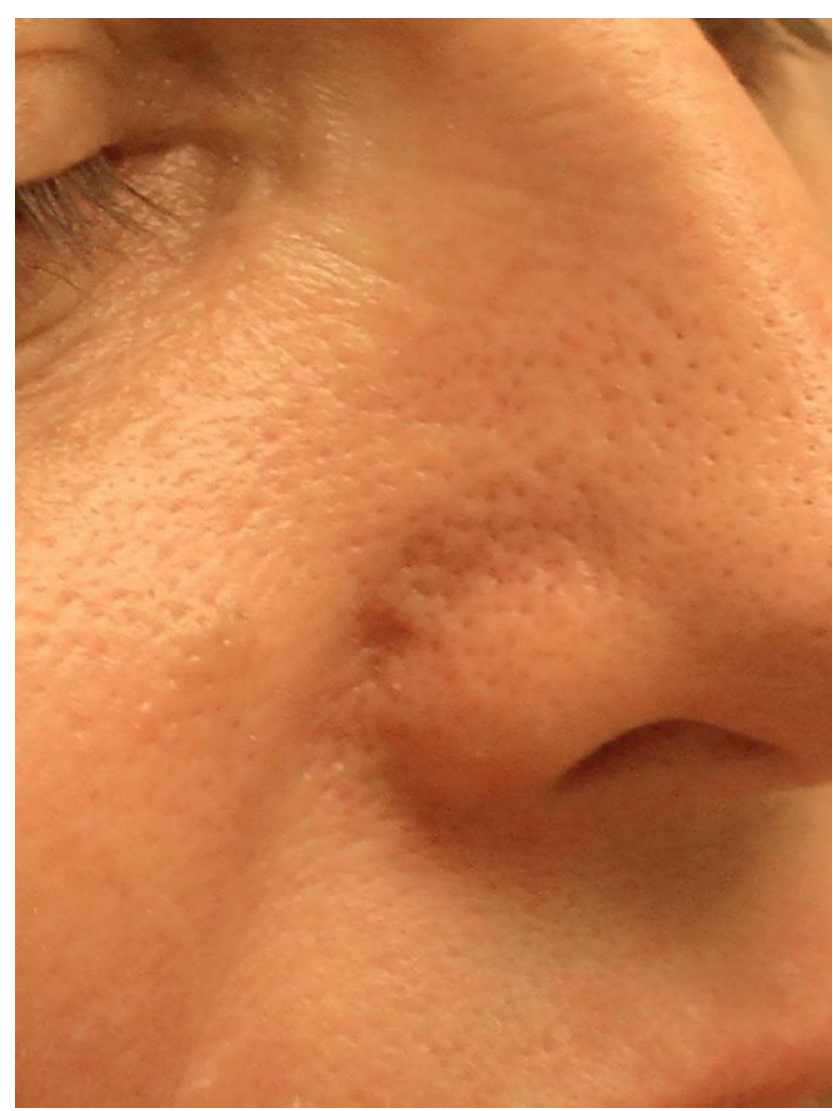

Figure 2 Dermal defect present on the alar groove following treatment with LP 1064 nm ND:YAG. Figure courtesy of Dr. Goldman.

The Nd:YAG laser is particularly good at treating unwanted larger facial veins. Use of a long-pulsed setting allows slow and uniform heating of veins causing endothelial damage and coagulation without vessel rupture or purpura. ${ }^{41}$ The lead author (MPG) commonly uses longpulsed 1064nm Nd:YAG lasers for treatment of facial reticular veins sized $1-3 \mathrm{~mm}$ in the temple and periorbital areas. Eremia and Li first demonstrated the safe and efficacious use of the $1064 \mathrm{~nm} \mathrm{Nd:YAG} \mathrm{in} \mathrm{the} \mathrm{treatment} \mathrm{of}$ blue venules and reticular facial veins. ${ }^{42}$ Bevin et al also demonstrated in eight patients the ability to treat facial vessels with diameters ranging in size between 0.3 and $2.0 \mathrm{~mm}$ in one treatment session. ${ }^{34}$ Half the subjects demonstrated a $26-50 \%$ vessel clearance and the other half demonstrated a $51-75 \%$ clearance rate. ${ }^{34}$ Longer pulses in the range of $20-60 \mathrm{~ms}$ durations ultimately achieved superior vessel clearance with minimal purpura compared to a pulse duration of $3 \mathrm{~ms}$ which invariably resulted in significant purpura. $^{34}$ Similarly, Lai and Goldman, demonstrated nearly $100 \%$ objective and subjective improvement in facial reticular veins in the temple and periorbital areas. ${ }^{43}$ Only 2 out of 20 patients treated in this study required a second laser session 6-12 months after the initial treatment. ${ }^{43}$ The authors note that rechanneling of existing veins can arise in the treatment zone necessitating re-treatment. ${ }^{43}$ Similar results were demonstrated in a large retrospective review by Aleisa and Goldman for facial reticular veins $1-3 \mathrm{~mm}$ in diameter. ${ }^{35}$

One common sequela of veins in the infraorbital region is dark circles. There are numerous causes of infraorbital dark circles so an appropriate evaluation is warranted as not all causes can or should be treated with lasers. Ma et al demonstrated the successful use of a long-pulsed 1064nm Nd:YAG laser for the eradication of visible infraorbital veins causing dark circles. ${ }^{44}$ In this study patients received 1-3 treatment sessions (mean of 1.6) with complete clearance that lasted 12 months after the last treatment. ${ }^{44}$ Treatments were very well tolerated, with post-treatment erythema and edema, the most frequently encountered adverse effect that resolved within days. ${ }^{44}$ The authors note that this laser can also induce new collagen formation which can improve skin texture, wrinkles, and laxity, at least to some extent, which can further improve the appearance of the infraorbital area. ${ }^{44}$ Treatment of periocular blue reticular veins have also been treated using this method with long-term results. ${ }^{45}$

One limitation of the Nd:YAG laser, especially for larger vessels that require higher fluences, is the pain described by some patients. If pain becomes a limiting factor for treatment, the use of topical anesthetic has been demonstrated to be effective without a decrease in efficacy of the laser treatment nor an alteration in potential complications or side effects from Nd:YAG laser treatments. ${ }^{46}$

\section{Picosecond Lasers}

Picosecond lasers deliver pulse durations in the picosecond range, much shorter, depending on the specifications of the individual device, than traditional nanosecond Q-switched lasers. ${ }^{47}$ Additionally, picosecond lasers deliver lower fluences allowing these devices to generate a greater photoacoustic effect with less photothermal damage. ${ }^{47}$ Wen et al demonstrated a statistically significant reduction in erythema using the Clinician Erythema Assessment Scale (CEAS) as well as a significant improvement in acne scars with minimal side effects in a split-face randomized study investigating the use of a $755 \mathrm{~nm}$ picosecond Alexandrite laser for the treatment of post-inflammatory erythema and acne scarring. ${ }^{47}$ 
Further studies investigating the role of picosecond lasers in the management of facial erythema are needed before reaching a conclusion regarding its utility in management of this common condition.

\section{Fractionated Lasers}

The main target of $1550 \mathrm{~nm}$ erbium-doped fractionated laser is water and it is typically utilized in skin rejuvenation procedures to aid in collagen remodeling. This laser can reach depths of approximately 1000 micrometers $(\mu \mathrm{m})$ where dermal blood vessels are located. ${ }^{48}$ Glaich et al demonstrated use of this laser in treatment of postinflammatory erythema secondary to acne vulgaris in one treatment. ${ }^{48}$ The authors contend that since water is a major component of blood vessels, this laser may lead to photothermal microvascular destruction leading to improvement in erythema. ${ }^{48}$ Park et al compared the use of a non-ablative $1550 \mathrm{~nm}$ fractionated laser with a $595 \mathrm{~nm}$ PDL for treatment of erythema secondary to acne and found that both lasers were effective in improvement of erythema with no statistically significant difference in results between the modalities. ${ }^{49}$ The authors hypothesize that the $1550 \mathrm{~nm}$ laser was effective because the primary pathology was a scar with erythematous components and not a primary vascular lesion nor erythema of the superficial portions of the skin. ${ }^{49}$ While we have not noticed an appreciable improvement in erythema using the 1550 or $1565 \mathrm{~nm}$ fractionated non-ablative lasers they may be an option for patients with photoaged skin, scars, and who want subtle improvements in skin laxity/wrinkling in addition to improvement in erythema.

\section{Other Light- and Energy-Based Devices Intense Pulsed Light (IPL)}

IPL is a non-coherent, broadband-filtered white light flashlamp technology that emits a continuous spectrum in the range of $500-1200 \mathrm{~nm} .^{20,21}$ Since this device emits a variety of wavelengths it has the capability of targeting a variety of chromophores including hemoglobin, melanin, and water with the use of the appropriate cut-off filters. $^{21,50}$ This device must be used with caution in patients who have a darker complexion or those who are $\tan$. In these darker patients the use of an IPL with multiple sequential pulsing utilizing a $30-40 \mathrm{~ms}$ delay between pulses is recommended for safe treatment. IPL should not be used where one does not want hair removed. The practitioner must hone their technique to ensure good contact of the sapphire cooling tip with the skin surface but also not be overly aggressive with applied pressure as these components can alter the result dramatically. ${ }^{50}$ Concomitant cold air cooling is also recommended both to decrease pain and minimize epidermal heating. ${ }^{51,52}$

Mark et al demonstrated the use of IPL with a $515 \mathrm{~nm}$ filter was able to decrease blood flow in areas of facial erythema, secondary to rosacea, by $30 \%$ using a scanning laser Doppler and a $21 \%$ decrease in the intensity of erythema. ${ }^{53}$ Tirico et al illustrated, in a randomized splitface study comparing IPL with PDL, a $60 \%$ and $45 \%$ reduction in facial redness in patients treated with shortpulse IPL and PDL, respectively. ${ }^{50}$ These results, however, did not meet significance. ${ }^{50}$ Similarly, Neuhaus et al illustrated, in a randomized study comparing the use of nonpurpuric PDL with IPL for treatment of facial erythema secondary to rosacea, that both devices were statistically significant in improving erythema and telangiectasias; however, there was no statistically significant difference between the two devices. ${ }^{24}$ Ultimately, Neuhaus et al determined that either PDL or IPL are efficacious for the improvement of erythema and telangiectasias and both had a tolerable side effect profile. ${ }^{24}$

A variety of studies have demonstrated the reduction of Demodex densities following IPL treatment. ${ }^{54-56}$ Prieto et al demonstrated, through both histologic and electron microscopic methods, a marked decrease in Demodex density as well as perifollicular inflammation correlating clinically with a reduction in erythema following IPL treatment. ${ }^{54}$ The authors also demonstrate that there is induction of coagulation necrosis of Demodex with preservation of the surrounding hair follicle. ${ }^{54}$ The hypothesis for the destruction of the mite but preservation of the follicle is the possibility of a specific chromophore that the mite contains which is more sensitive to the IPL energy than the hair follicle. ${ }^{54}$ It is also proposed that the spherical shape of the Demodex mite does not transfer energy as efficiently as cylindrically shaped hair follicles, thus also helping to preserve the follicular unit. ${ }^{54}$ Fishman et al provided further evidence of the eradication of Demodex mites with use of IPL through realtime in-vitro video microscopy. ${ }^{55}$ In one case report, it was suggested that Demodex, which are light and heat sensitive, can incite an inflammatory response when IPL is used thereby worsening one's rosacea. ${ }^{57}$ Paradoxical worsening of rosacea after use of IPL has not been the experience in our practice where IPL is one of our go-to treatment modalities. 
We typically use a $560 \mathrm{~nm}$ cutoff filter with a double pulse of $3.0 \mathrm{~ms}$ and $3.0 \mathrm{~ms}$, with $10-20 \mathrm{msec}$ delay at 17 $19 \mathrm{~J} / \mathrm{cm}^{2}$ with the Stellar M22 (Lumenis, Yokneam, Israel). We encourage patients to undergo maintenance treatments at least twice yearly with IPL to help prevent flares of rosacea (Figures 3 and 4).

\section{Photodynamic Therapy (PDT)}

Photodynamic therapy (PDT) uses a photosensitizing agent, an activating light source, and oxygen to treat a variety of cutaneous diseases including actinic keratoses, superficial skin cancers, generalized photodamage, acne vulgaris, and even rosacea. ${ }^{58}$ The two photosensitizing agents commonly applied to the treatment area are either 5-aminolevulinic acid (ALA) or the more lipophilic methylaminolevulinic acid (MAL) ${ }^{58}$ Both topical agents are absorbed by the sebaceous glands and subsequently metabolized to produce the highly photoactive agent, protoporphyrin IX (PpIX). ${ }^{58}$ Excitation of PpIX with a light source of the appropriate wavelength will result in cytotoxic singlet oxygen species which will then cause localized oxidative stress and targeted cell death. ${ }^{58}$ Photoactivation of porphyrins can be accomplished with a variety of light and laser sources including incoherent, continuous-wave red or blue light, PDL, or IPL. ${ }^{58}$ Since the absorption spectrum of PpIX includes both a maximal peak at 410nm (known as the Soret band)

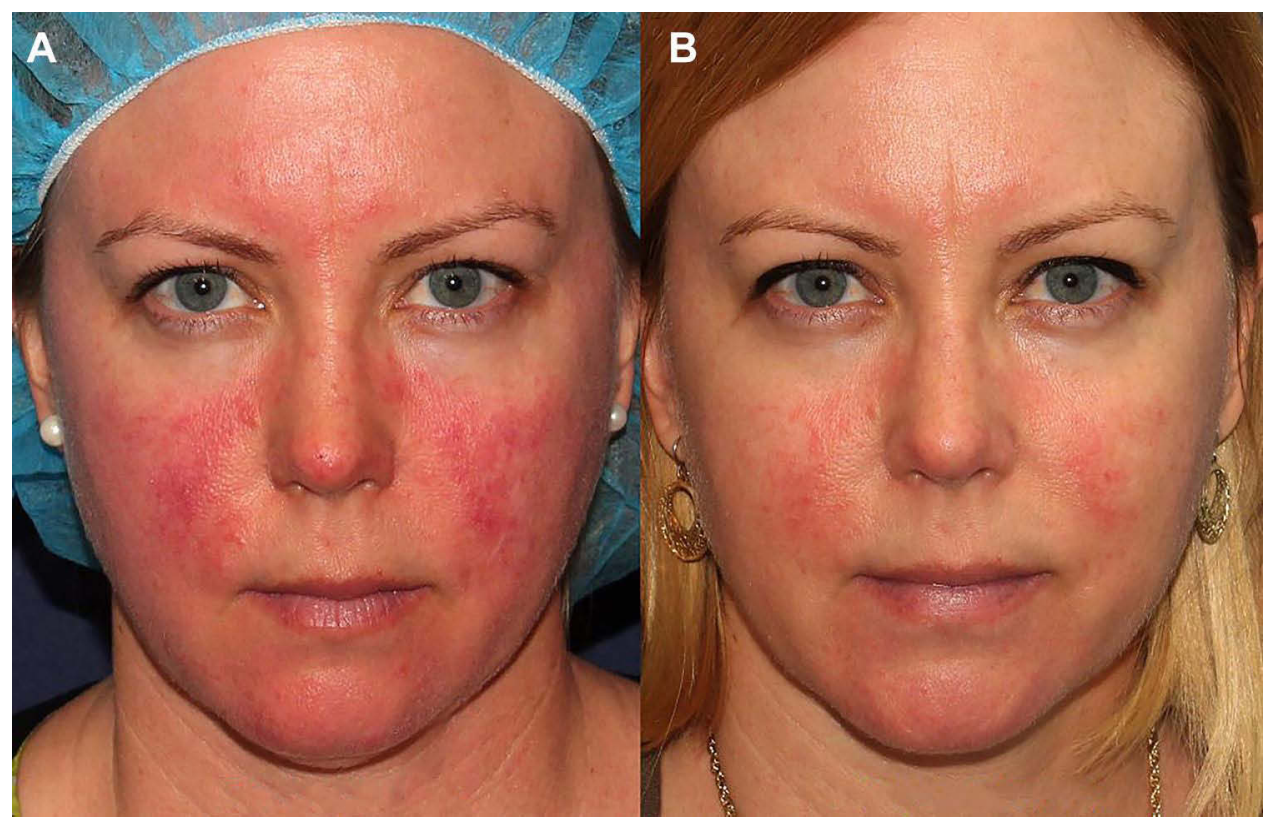

Figure 3 Before $(\mathbf{A})$ and after $(\mathbf{B})$ one treatment with IPL for rosacea. Figure courtesy of Dr. Goldman.

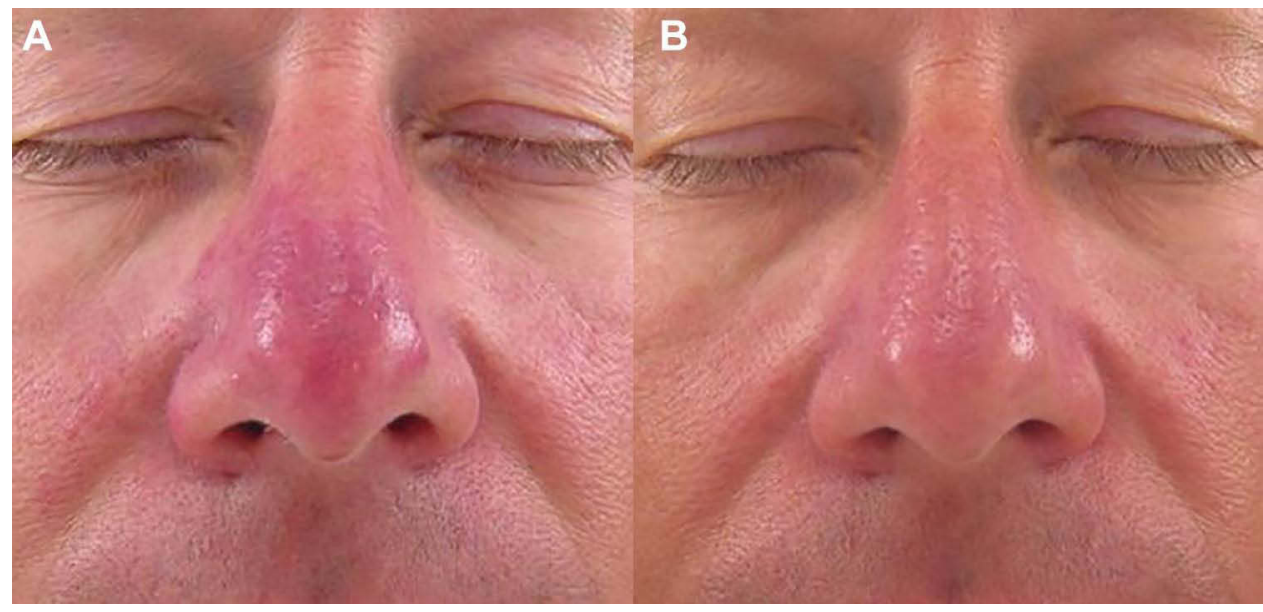

Figure 4 Before (A) and after (B) IPL treatment of red nose rosacea with Lumenis One (20 J/cm2, 3.5-3.5 msec). Figure courtesy of Dr. Goldman. 
as well as four smaller peaks between $500-630 \mathrm{~nm}(\mathrm{Q}$ bands), there is a lot of versatility in choosing an activating light source and even combining light/laser sources for optimized results. ${ }^{58}$ In fact, the practitioner can use blue light which has anti-inflammatory and epidermal turnover properties in combination with red light which penetrates deeper and has both antimicrobial properties and can even focally target sebaceous glands for destruction (Figure 5). ${ }^{58}$ Additionally, using different light/laser sources will enable one to activate all the specific absorption peaks of PpIX allowing for maximal photobleaching and improved overall efficacy. $^{58}$

Friedmann et al demonstrated mild-to-moderate improvement in rosacea and moderate improvement in skin quality in a 30-patient retrospective study following treatment with ALA-PDT in a variety of treatment scenarios including: blue light + PDL; blue light + IPL; blue light + PDL + IPL; or blue light + red light + PDL + IPL. ${ }^{58}$ Patients treated with PDL and/or IPL had these treatments performed prior to the PDT. ${ }^{58}$ Although this study did not show statistically significant differences in the results with use of multiple, sequential modalities, this study does demonstrate the safety of using multiple light/laser devices in a single treatment session (Figure 6). ${ }^{58}$ Furthermore, there was no statistically significant difference in patient-reported adverse effects including post-procedure erythema, pain, or acne flares. ${ }^{58}$ The authors suggest that PDT improves rosacea by elimination of Demodex mites through PDT-mediated cytotoxic reactive oxygen species and amelioration of excessive glandular components of the disease process through direct photodynamic injury to hyperplastic and inflamed pilosebaceous units. ${ }^{58}$ Additionally, it has been suggested that PDT has the ability to modulate the immune system and induce apoptosis of $\mathrm{T}$ cells which can also help ameliorate rosacea. $^{59}$

Katz and Patel demonstrated the use of ALA-PDT with PDL activation for recalcitrant rosacea that showed improvement after the second treatment and sustained improvement after the six treatments completed every two weeks. ${ }^{60}$ Fan et al treated 20 patients with erythematotelangiectatic or papulopustular rosacea and Fitzpatrick skin types III and IV with ALA-PDT activated with LED red light for four sessions every ten days. ${ }^{59}$ The authors were able to demonstrate a statistically significant clinical improvement in flushing, erythema, and telangiectasias compared to baseline as well as clearance of all inflammatory lesions in all patients after 24 weeks. ${ }^{59}$ The authors demonstrated a reduction of flushing, erythema, and inflammatory lesion count of $87.1 \%, 89.5 \%$, and $97.2 \%$, respectively from baseline through the last follow-up visit. ${ }^{59}$ Finally, $65 \%$ and $25 \%$ of patients rated their satisfaction after the 4 treatments as very satisfied and satisfied, respectively. ${ }^{59}$

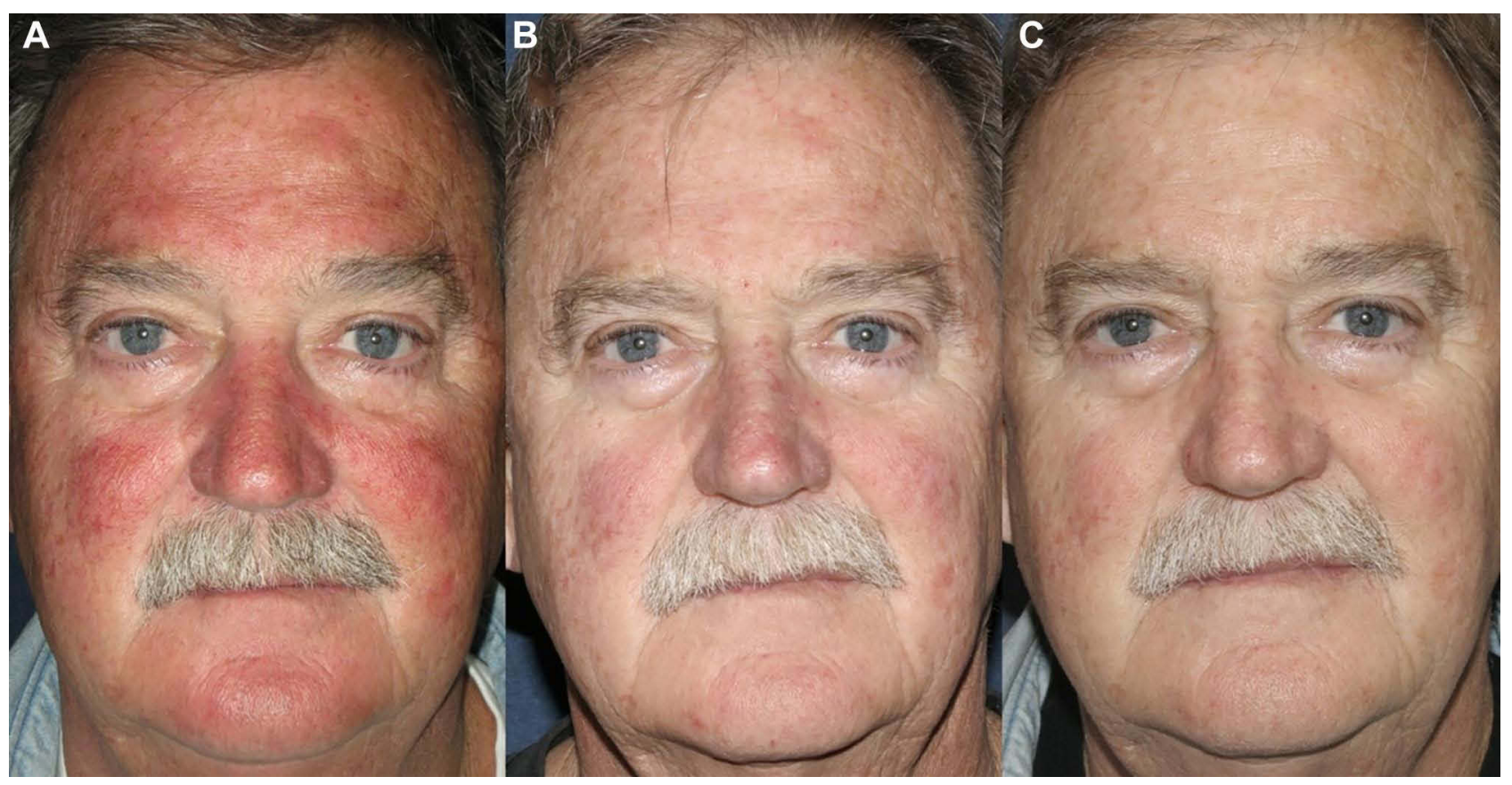

Figure 5 PDT blue light for rosacea, pre-treatment (A), post I treatment (B), post two treatments (C). Figure courtesy of Dr. Goldman. 


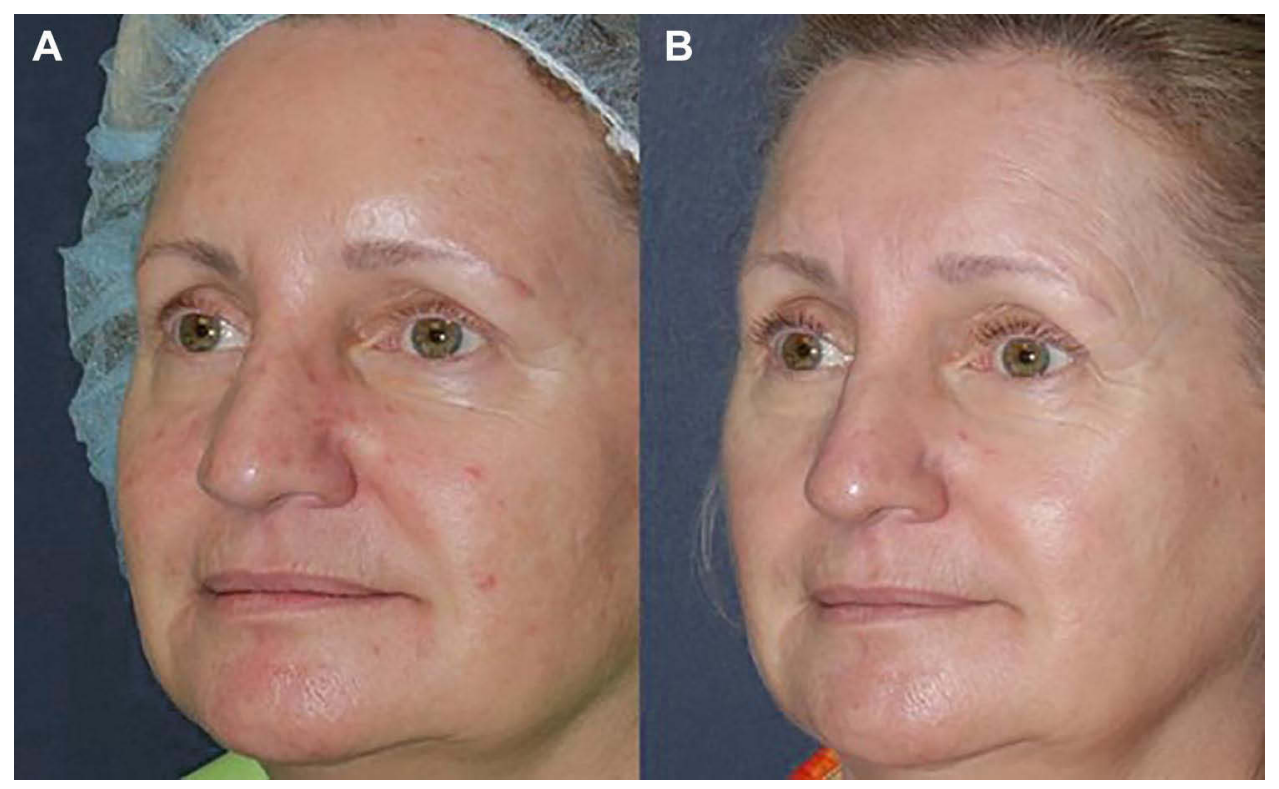

Figure 6 Before (A) and after (B) ALA-PDT for treatment of rosacea with PDL, IPL, and blue/red light. Figure courtesy of Dr. Goldman.

In a retrospective study investigating MAL-PDT for off-label use in a variety of inflammatory and aesthetic cutaneous conditions in 20 different Italian dermatology departments, Calzavara-Pinton et al demonstrated 6 out of 7 patients with rosacea had a marked or moderate improvement and all patients had an excellent cosmetic outcome. ${ }^{61}$ Additionally, it was disclosed that only one patient with rosacea relapsed during the study period. ${ }^{61}$ In a small case series, Nybaek and Jemec treated 4 patients with rosacea with MAL-PDT activated by a $632 \mathrm{~nm}$ red diode light and demonstrated 3 out of 4 patients experienced remission of their rosacea for 3 months or longer after $2-3$ PDT treatments. ${ }^{62}$ In a larger follow-up study, Bryld and Jemec treated 17 patients presenting with rosacea with MAL-PDT activated with red light and 10 patients were reported to have drastic relief of rosacea symptoms without the need for adjuvant therapy. ${ }^{63}$ The authors noted that PDT in their practice was better at treating the papulopustular variant than other types of rosacea. ${ }^{63}$

PDT can be a reasonable treatment option for rosacea when patients are unresponsive or recalcitrant to traditional therapies. Moreover, PDT should be considered for patients presenting with actinic keratoses and diffuse photodamage in addition to erythema secondary to rosacea (Figure 5).

\section{Radiofrequency Microneedling}

Fractional radiofrequency microneedling (RFRM) is a newer technology that delivers heat via radiofrequency energy to the dermis through an array of multiple $30-32 \mathrm{G}$ needles without damaging the overlying epidermis. ${ }^{64}$ Radiofrequency devices use electromagnetic radiation that is converted to thermal energy producing controlled dermal damage which subsequently hastens healing and new collagen formation. ${ }^{65}$ Numerous companies make RFRM devices, all with different tunable variables such as number of needles, insulated versus non-insulated needles, energy level, and conduction time. ${ }^{66}$ The density of the treatment is determined by the practitioner by the number of passes that are completed. This versatility gives the practitioner a variety of options to customize a treatment to an individual patient's condition and skin type. Since this modality does not damage the epidermis, it offers patients quick recovery times and is particularly good for treating skin of color patients. ${ }^{64,67}$ Additionally, this technology induces both neo-collagenesis as well as neo-elastogenesis helping to improve skin laxity and wrinkles, which can be a primary or secondary benefit depending on the indication for which this instrument is used. $^{64,67}$

RFRM has yet to be validated for the treatment of rosacea and/or diffuse erythema; however, a number of 
small studies have reported at least modest benefit. ${ }^{64,67}$ Park et al demonstrated improvement in rosacea and erythema after two treatments of RFRM with the reduction in erythema more effective in those with the papulopustular subtype. ${ }^{64}$ They also noted patient satisfaction regarding self-assessment of rosacea symptoms and flushing. ${ }^{64}$ Min et al demonstrated similar results after using RFRM for treatment of acne-related post-inflammatory erythema. ${ }^{67}$ Interestingly, it has been demonstrated through histologic and immunohistochemistry staining analysis, that there is a reduction of inflammation and angiogenesis after RFRM treatments. ${ }^{64,67}$ It should be highlighted that patients will have mild erythema up to a few days after the treatment. Although this treatment option for rosacea and/or erythema has not been widely validated, RFRM is possibly another tool that can be added to our armamentarium.

\section{Other Treatment Modalities}

One should take extreme caution when attempting to use sclerotherapy to treat telangiectasias or other visible fine vessels on the face, in what some practitioners call "microsclerotherapy". This is a less predictable and less reliable treatment that can cause hyperpigmentation, ulceration, and scarring. ${ }^{68,69}$ Furthermore, the high vascularity of the face with innumerable valveless anastomoses and close proximity to the facial artery, among other named vessels, makes this treatment method less than ideal when there are many other better, safer, and more predictable options. ${ }^{70}$ Unintended blindness is a possibility if the sclerosing agent enters the periocular vessels. ${ }^{35}$ Finally, removal of dilated periocular veins by phlebectomy should only be considered by those with special training for this procedure and when less invasive techniques are not possible. ${ }^{70}$

\section{Conclusion}

There are numerous medical and procedural treatment options for patients with unwanted facial erythema, telangiectasias, and/or reticular veins. Determining the etiology of facial erythema and patients' goals are imperative. Furthermore, the need for combination treatments to achieve the desired result must to be recognized.

\section{Photo Consent}

All the individuals who appear in the figures contained in our manuscript provided informed consent for their images to be published.

\section{Disclosures}

Dr Mitchel Goldman performs research for Lumenis and reported no other potential conflicts of interest for this work. Drs Loyal, Carr, and Almukhtar reported no conflicts of interest for this work.

\section{References}

1. Gether L, Overgaard LK, Egeberg A, et al. Incidence and prevalence of rosacea: a systematic review and meta-analysis. $\mathrm{Br} J$ Dermatol. 2018;179(2):282-289.

2. Moustafa F, Lewallen RS, Feldman SR. The psychological impact of rosacea and the influence of current management options. $\mathrm{J} \mathrm{Am} \mathrm{Acad}$ Dermatol. 2014;71(5):973-980. doi:10.1016/j.jaad.2014.05.036

3. Del Rosso JQ. Advances in understanding and managing rosacea: part 2: the central role, evaluation, and medical management of diffuse and persistent facial erythema of rosacea. $J$ Clin Aesthet Dermatol. 2012;5(3):26-36.

4. Jackson JM, Knuckles M, Minni JP, et al. The role of brimonidine tartrate gel in the treatment of rosacea. Clin Cosmet Investig Dermatol. 2015;8:529-538. doi:10.2147/CCID.S58920

5. Chunharas C, Boen M, Alhaddad M, et al. The efficacy of pulsed dye laser pretreated with or without local anesthetic on patients presenting with erythema of face, neck, chest, and extremities. Lasers Surg Med. 2020;52(4):307-314. doi:10.1002/1sm.23146

6. Anderson MS, Nadkarni A, Cardwell LA, et al. Spotlight on brimonidine topical gel $0.33 \%$ for facial erythema of rosacea: safety, efficacy, and patient acceptability. Patient Prefer Adherence. 2017;11:1143-1150. doi:10.2147/PPA.S115708

7. Cline A, McGregor SP, Feldman SR. Medical management of facial redness in rosacea. Dermatol Clin. 2018;36(2):151-159. doi:10.1016/ j.det.2017.11.010

8. Vissing A-CE, Dierickx C, Karmisholt KE, et al. Topical brimonidine reduces IPL-induced erythema without affecting efficacy: a randomized controlled trial in patients with facial telangiectasias. Lasers Surg Med. 2018;50(10):1002-1009. doi:10.1002/1sm.22953

9. Lowe E, Lim S. Paradoxical erythema reaction of long-term topical brimonidine gel for the treatment of facial erythema of rosacea. J Drugs Dermatol. 2016;15(6):763-765.

10. Hoover RM, Erramouspe J. Role of topical oxymetazoline for management of erythematotelangiectatic rosacea. Ann Pharmacother. 2018;52(3):263-267. doi:10.1177/1060028017740139

11. Patel NU, Shukla S, Zaki J, Feldman SR. Oxymetazoline hydrochloride cream for facial erythema associated with rosacea. Expert Rev Clin Pharmacol. 2017;10(10):1049-1054. doi:10.1080/ 17512433.2017.1370370

12. Tanghetti EA, Goldberg DJ, Dover JS, et al. Oxymetazoline and energy-based therapy in patients with rosacea: evaluation of the safety and tolerability in an Open-Label, Interventional Study. Lasers Surg Med. 2021;53(1):55-65. doi:10.1002/1sm.23253

13. Suggs AK, Macri A, Richmond H, et al. Treatment of erythematotelangiectatic rosacea with pulsed-dye laser and oxymetazoline $1.0 \%$ Cream: a Retrospective Study. Lasers Surg Med. 2020;52(1):38-43. doi: $10.1002 / 1 \mathrm{sm} .23176$

14. Okwundu N, Cline A, Feldman SR. Difference in vasoconstrictors: oxymetazoline vs. brimonidine. J Dermatolog Treat. 2019;1-7.

15. Yuraitis M, Jacob CI. Botulinum toxin for the treatment of facial flushing. Dermatol Surg. 2004;30(1):102-104. doi:10.1111/j.15244725.2004.30017.x

16. Al-Niaimi F, Glagoleva E, Araviiskaia E. Pulsed dye laser followed by intradermal botulinum toxin type-A in the treatment of rosacea-associated erythema and flushing. Dermatol Ther. 2020;33 (6):e13976. doi:10.1111/dth.13976 
17. Bloom BS, Payongayong L, Mourin A, et al. Impact of intradermal abobotulinumtoxinA on facial erythema of rosacea. Dermatol Surg. 2015;41(Suppl 1):S9-S16. doi:10.1097/DSS.0000000000000277

18. Sterodimas A, Nicolaou M, Paes TR. Successful use of botulinum toxin-A for the treatment of neck and anterior chest wall flushing. Clin Exp Dermatol. 2003;28(6):592-594. doi:10.1046/j.13652230.2003.01379.x

19. Khoury JG, Saluja R, Goldman MP. The effect of botulinum toxin type A on full-face intense pulsed light treatment: a randomized, double-blind, split-face study. Dermatol Surg. 2008;34 (8):1062-1069. doi:10.1111/j.1524-4725.2008.34207.x

20. Tan SR, Tope WD. Pulsed dye laser treatment of rosacea improves erythema, symptomatology, and quality of life. J Am Acad Dermatol. 2004;51(4):592-599. doi:10.1016/j.jaad.2004.04.010

21. Laube S, Lanigan SW. Laser treatment of rosacea. J Cosmet Dermatol. 2002;1(4):188-195. doi:10.1111/j.1473-2165.2002.00071.x

22. Hofmann MA, Lehmann P. Physical modalities for the treatment of rosacea. J Dtsch Dermatol Ges. 2016;14(Suppl 6):38-43.

23. Uebelhoer NS, Bogle MA, Stewart B, et al. A split-face comparison study of pulsed 532-nm KTP laser and 595-nm pulsed dye laser in the treatment of facial telangiectasias and diffuse telangiectatic facial erythema. Dermatol Surg. 2007;33(4):441-448. doi:10.1111/j.15244725.2007.33091.x

24. Neuhaus IM, Zane LT, Tope WD. Comparative efficacy of nonpurpuragenic pulsed dye laser and intense pulsed light for erythematotelangiectatic rosacea. Dermatol Surg. 2009;35(6):920-928. doi:10.1111/j.1524-4725.2009.01156.x

25. Iyer S, Fitzpatrick RE. Long-pulsed dye laser treatment for facial telangiectasias and erythema: evaluation of a single purpuric pass versus multiple subpurpuric passes. Dermatol Surg. 2005;31(8 Pt 1):898-903. doi:10.1097/00042728-200508000-00002

26. Becher GL, Cameron H, Moseley H. Treatment of superficial vascular lesions with the KTP 532-nm laser: experience with 647 patients Lasers Med Sci. 2014;29(1):267-271. doi:10.1007/s10103-013-1330-5

27. Clark SM, Lanigan SW, Marks R. Laser treatment of erythema and telangiectasia associated with rosacea. Lasers Med Sci. 2002;17 (1):26-33. doi:10.1007/s10103-002-8263-8

28. Bulbul Baskan E, Akin Belli A. Evaluation of long-term efficacy, safety, and effect on life quality of pulsed dye laser in rosacea patients. J Cosmet Laser Ther. 2019;21(4):185-189. doi:10.1080/ 14764172.2018.1502453

29. Alam M, Voravutinon N, Warycha M, et al. Comparative effectiveness of nonpurpuragenic 595-nm pulsed dye laser and microsecond 1064-nm neodymium: yttrium-aluminum-garnetlaser for treatment of diffuse facial erythema: a double-blind randomized controlled trial. $J$ Am Acad Dermatol. 2013;69(3):438-443. doi:10.1016/j. jaad.2013.04.015

30. Ertaş R, Yaman O, Akkuş MR, et al. The rapid effect of pulsed dye laser on demodex density of facial skin. J Cosmet Laser Ther. 2019;21(3):123-126. doi:10.1080/14764172.2018.1481509

31. Keaney TC, Tanzi E, Alster T. Comparison of $532 \mathrm{~nm}$ potassium titanyl phosphate laser and $595 \mathrm{~nm}$ pulsed dye laser in the treatment of erythematous surgical scars: a Randomized, Controlled, Open-Label Study. Dermatol Surg. 2016;42(1):70-76. doi:10.1097/ DSS.0000000000000582

32. Asiran Serdar Z, Fisek Izci N. The evaluation of long-pulsed Nd: YAGlaser efficacy and side effects in the treatment of cutaneous vessels on the face and legs. J Cosmet Dermatol. 2020;19 (7):1656-1661. doi:10.1111/jocd.13208

33. Kwon WJ, Park BW, Cho EB, et al. Comparison of efficacy between long-pulsed Nd:YAG laser and pulsed dye laser to treat rosacea-associated nasal telangiectasia. $J$ Cosmet Laser Ther. 2018;20(5):260-264. doi:10.1080/14764172.2017.1418510
34. Bevin AA, Parlette EC, Domankevitz Y, et al. Variable-pulse Nd: YAGlaser in the treatment of facial telangiectasias. Dermatol Surg. 2006;32(1):7-12. doi:10.1097/00042728-200601000-00002

35. Aleisa A, Goldman MP. A dynamically cooled, variable spot-sized $1064 \mathrm{~nm}$ neodymium-doped yttrium aluminum garnet laser as a treatment option for facial reticular veins. Dermatol Surg. 2021;47(1):76-78. doi:10.1097/DSS.0000000000002406

36. Salem SAM, Abdel Fattah NS, Tantawy SM, et al. Neodymiumyttrium aluminum garnet laser versus pulsed dye laser in erythemato-telangiectatic rosacea: comparison of clinical efficacy and effect on cutaneous substance (P) expression. J Cosmet Dermatol. 2013;12(3):187-194. doi:10.1111/jocd.12048

37. Campos MA, Sousa AC, Varela P, et al. Comparative effectiveness of purpuragenic $595 \mathrm{~nm}$ pulsed dye laser versus sequential emission of $595 \mathrm{~nm}$ pulsed dye laser and $1064 \mathrm{~nm}$ Nd:YAG laser: a double-blind randomized controlled study. Acta Dermatovenerol Alp Pannonica Adriat. 2019;28(1):1-5.

38. Karsai S, Roos S, Raulin C. Treatment of facial telangiectasia using a dual-wavelength laser system (595 and $1064 \mathrm{~nm}$ ): a randomized controlled trial with blinded response evaluation. Dermatol Surg. 2008;34(5):702-708. doi:10.1111/j.1524-4725.2008.34131.x

39. Lee JH, Na SY, Choi M, et al. Long-pulsed Nd: YAG laser: does it give clinical benefit on the treatment of resistant telangiectasia? J Eur Acad Dermatol Venereol. 2012;26(10):1280-1284. doi:10.1111/ j.1468-3083.2011.04282.x

40. Yalici-Armagan B, Elcin G. The decrease of demodex density after $\mathrm{Nd}$ : YAGlaser application for facial telengiactasias: a case report. Dermatol Ther. 2020;33(6):e14108. doi:10.1111/dth.14108

41. Lee TS, Kwek JWM, Ellis DAF. Treatment of periocular and temporal reticular veins with 1064-nm Nd:YAG Laser. J Cosmet Dermatol. 2020;19(9):2306-2312. doi:10.1111/jocd.13504

42. Eremia S, Li CY. Treatment of face veins with a cryogen spray variable pulse width $1064 \mathrm{~nm}$ Nd: YAGLaser: a prospective study of 17 patients. Dermatol Surg. 2002;28(3):244-247. doi:10.1046/ j.1524-4725.2002.01217.x

43. Lai SW, Goldman MP. Treatment of facial reticular veins with dynamically cooled, variable spot-sized $1064 \mathrm{~nm}$ Nd: YAGlaser. J Cosmet Dermatol. 2007;6(1):6-8. doi:10.1111/j.1473-2165.2007.00256.x

44. Ma G, Lin -X-X, Hu X-J, et al. Treatment of venous infraorbital dark circles using a long-pulsed 1064-nm neodymium-doped yttrium aluminum garnet laser. Dermatol Surg. 2012;38(8):1277-1282. doi:10.1111/j.1524-4725.2012.02457.x

45. Goldman A, Lotti T, Tchernev G, et al. Successful treatment of reticular blue veins of the lower eyelid by long-pulse Nd: YAG case report with 8-year follow-up. Open Access Maced J Med Sci. 2017;6(1):58-60. doi:10.3889/oamjms.2018.021

46. Bonaparte JP, Ellis DE. Discomfort during periorbital and lateral temporal laser vein treatment: a double-blind randomized controlled trial. Plast Reconstr Surg Glob Open. 2014;2(5):e159. doi:10.1097/ GOX.0000000000000106

47. Wen X, Li Y, Hamblin MR, et al. A randomized split-face, investigator-blinded study of a picosecond Alexandrite laser for post-inflammatory erythema and acne scars. Dermatol Ther. 2020;33(6):e13941. doi:10.1111/dth.13941

48. Glaich AS, Goldberg LH, Friedman RH, et al. Fractional photothermolysis for the treatment of postinflammatory erythema resulting from acne vulgaris. Dermatol Surg. 2007;33(7):842-846. doi:10.1111/j.1524-4725.2007.33180.x

49. Park KY, Ko EJ, Seo SJ, et al. Comparison of fractional, nonablative, 1550-nm laser and 595-nm pulsed dye laser for the treatment of facial erythema resulting from acne: a split-face, evaluator-blinded, randomized pilot study. J Cosmet Laser Ther. 2014;16(3):120-123. doi:10.3109/14764172.2013.854626 
50. Tirico MCCP, Jensen D, Green C, et al. Short pulse intense pulsed light versus pulsed dye laser for the treatment of facial redness. J Cosmet Laser Ther. 2020;22(2):60-64. doi:10.1080/ 14764172.2020 .1717540

51. Wat H, Wu DC, Rao J, Goldman MP. Application of intense pulsed light in the treatment of dermatologic disease: a systematic review. Dermatol Surg. 2014;40(4):359-377. doi:10.1111/dsu.12424

52. Almukhtar R, Carr E, Goldman M. Intense pulsed light: the early years. Dermatol Rev. 2020;1-10.

53. Mark KA, Sparacio RM, Voigt A, et al. Objective and quantitative improvement of rosacea-associated erythema after intense pulsed light treatment. Dermatol Surg. 2003;29(6):600-604. doi:10.1046/ j.1524-4725.2003.29141.x

54. Prieto VG, Sadick NS, Lloreta J, et al. Effects of intense pulsed light on sun-damaged human skin, routine, and ultrastructural analysis. Lasers Surg Med. 2002;30(2):82-85. doi:10.1002/1sm.10042

55. Fishman HA, Periman LM, Shah AA. Real-time video microscopy of in vitro demodex death by intense pulsed light. Photobiomodul Photomed Laser Surg. 2020;38(8):472-476. doi:10.1089/ photob.2019.4737

56. Zhang X, Song N, Gong L. Therapeutic effect of intense pulsed light on ocular demodicosis. Curr Eye Res. 2019;44(3):250-256. doi:10.1080/02713683.2018.1536217

57. Wang $\mathrm{P}$, Zhang L, Shi L, et al. Latent demodex infection contributes to intense pulsed light aggravated rosacea: cases serial. J Cosmet Laser Ther. 2019;21(3):163-165. doi:10.1080/14764172.20 18.1502448

58. Friedmann DP, Goldman MP, Fabi SG, et al. Multiple sequential light and laser sources to activate aminolevulinic acid for rosacea. J Cosmet Dermatol. 2016;15(4):407-412. doi:10.1111/jocd.12231

59. Fan L, Yin R, Lan T, Hamblin MR. Photodynamic therapy for rosacea in Chinese patients. Photodiagnosis Photodyn Ther. 2018;24:82-87. doi:10.1016/j.pdpdt.2018.08.005

60. Katz B, Patel V. Photodynamic therapy for the treatment of erythema, papules, pustules, and severe flushing consistent with rosacea. J Drugs Dermatol. 2006;5(2 Suppl):6-8.
61. Calzavara-Pinton PG, Rossi MT, Aronson E, et al. A retrospective analysis of real-life practice of off-label photodynamic therapy using methyl aminolevulinate (MAL-PDT) in 20 Italian dermatology departments. Part 1: inflammatory and aesthetic indications. Photochem Photobiol Sci. 2013;12(1):148-157. doi:10.1039/ c2pp25124h

62. Nybaek H, Jemec GBE. Photodynamic therapy in the treatment of rosacea. Dermatology. 2005;211(2):135-138. doi:10.1159/000086443

63. Bryld LE, Jemec GB. Photodynamic therapy in a series of rosacea patients. J Eur Acad Dermatol Venereol. 2007;21(9):1199-1202. doi:10.1111/j.1468-3083.2007.02219.x

64. Park SY, Kwon HH, Yoon JY, et al. Clinical and histologic effects of fractional microneedling radiofrequency treatment on rosacea. Dermatol Surg. 2016;42(12):1362-1369. doi:10.1097/DSS.0000000 000000888

65. Kim S-J, Lee Y, Seo Y-J, et al. Comparative efficacy of radiofrequency and pulsed dye laser in the treatment of rosacea. Dermatol Surg. 2017;43(2):204-209. doi:10.1097/DSS.0000000000000968

66. Chunharas C, Wu D, Goldman MP. Chapter 8: microneedling and radiofrequency. In: Hausauer $\mathrm{AK}$, Jones $\mathrm{DH}$, editors. $P R P$ and Microneedling in Aesthetic Medicine. New York, NY: Theime Medical Publishers, Inc.; 2019:70-84.

67. Min S, Park SY, Yoon JY, et al. Fractional microneedling radiofrequency treatment for acne-related post-inflammatory erythema. Acta Derm Venereol. 2016;96(1):87-91. doi:10.2340/00015555-2164

68. Kim Y-J, Shin B-S, Chung B-S, et al. A simple technique for treatment of nasal telangiectasia using trichloroacetic acid and $\mathrm{CO} 2$ laser. Dermatol Surg. 2002;28(8):729-731. doi:10.1046/j.15244725.2002.02001.x

69. Fante RG, Goldman MP. Removal of periocular veins by sclerotherapy. Ophthalmology. 2001;108(3):433-434. doi:10.1016/ S0161-6420(00)00565-0

70. Weiss RA, Ramelet -A-A. Removal of blue periocular lower eyelid veins by ambulatory phlebectomy. Dermatol Surg. 2002;28(1):43-45. doi:10.1046/j.1524-4725.2002.01189.x

\section{Publish your work in this journal}

Clinical, Cosmetic and Investigational Dermatology is an international, peer-reviewed, open access, online journal that focuses on the latest clinical and experimental research in all aspects of skin disease and cosmetic interventions. This journal is indexed on CAS.
The manuscript management system is completely online and includes a very quick and fair peer-review system, which is all easy to use. Visit http://www.dovepress.com/testimonials.php to read real quotes from published authors. 